\title{
ADAPTIVE PLASTICITY IN THE PRIMATE SPINAL STRETCH REFLEX: EVIDENCE FOR A TWO-PHASE PROCESS ${ }^{1}$
}

\author{
JONATHAN R. WOLPAW* ${ }^{*}$ AND JULIE A. O'KEEFE* \\ * Wadsworth Center for Laboratories and Research, New York State Department of Health, Albany, New York 12201 and \\ $*+$ Departments of Neurology and Anatomy, Albany Medical College, Albany, New York 12208
}

Received December 20, 1983; Revised May 24, 1984; Accepted May 30, 1984

\begin{abstract}
Monkeys can slowly increase or decrease the amplitude of the purely spinal, largely monosynaptic portion of the response to sudden muscle stretch, the spinal stretch reflex (SSR), when confronted by a task requiring such change (Wolpaw, J. R., V. A. Kieffer, R. F. Seegal, D. J. Braitman, and M. G. Sanders (1983) Brain Res. 267: 196-200; Wolpaw, J. R., D. J. Braitman, and R. F. Seegal (1983) J. Neurophysiol. 50: 1296-1311). Change occurs without alteration in initial muscle length or in background activity of agonist, antagonist, or synergist muscles. This study uses composite curves to describe in detail the development of SSR amplitude change. It reveals important, previously unexpected features of this development.

SSR increase or decrease appears to occur in two distinct phases. Phase I, a nearly immediate $8 \%$ change, occurs within the first $6 \mathrm{hr}$. Phase II, a $2 \%$ /day change, continues for at least 2 months. Although phase II is much slower than phase I, its final magnitude is far greater.

Phase I indicates a nearly immediate change in suprasegmental influence on the segmental arc of the SSR. Because stretch onset time is unpredictable and the SSR occurs before any other possible response, this change in descending activity must be tonic; it must be present continually, day after day, for the 5 to $7 \mathrm{hr} /$ day the animal spends at the task. Phase I produces a rapid and significant increase in reward probability. Thus, it may be readily interpreted as an example of operant conditioning, provoked by the reward contingency.

Phase II's extremely slow rate distinguishes it from phase I and suggests that, unlike phase I, phase II represents long-term plasticity, with a persistent structural or biochemical basis. This slow rate makes it difficult to view phase II as operantly conditioned: it seems much too slow to provide the animal with detectable reinforcement. Why, then, does it occur at all? One reasonable explanation is that phase II is a side effect of the change in tonic descending activity responsible for phase I. If, as related evidence suggests, part or all of this long-term alteration occurs at the segmental level, it should constitute a technically accessible substrate of memory.
\end{abstract}

Investigation of mechanisms of memory, or long-term adaptive change, in the primate CNS requires a stimulus-response pathway which satisfies three requirements. It must: (1) be defined and accessible anatomically and physiologically; (2) be capable of displaying long-term adaptive change; and (3) contain the responsible substrate. The initial, purely spinal, largely monosynaptic, portion of the response to sudden muscle stretch, the spinal stretch reflex (SSR), satisfies the first requirement. Several years ago, we began studies which have now demonstrated that it also satisfies the second requirement, capacity for adaptive change (Wolpaw, 1983; Wolpaw et al.,

\footnotetext{
${ }^{1}$ We thank Ms. Patricia A. Noonan for invaluable technical assistance, Dr. David L. Martin for aid in generation of the computer-fitted equations, Drs. Richard F. Seegal and Robert I. Dowman for reviewing the manuscript, and Ms. Joyce White for typing the manuscript. This work was supported in part by Grant R-322-82 from the United Cerebral Palsy Research and Educational Foundation.

${ }^{2}$ To whom correspondence should be addressed, at Wadsworth Center for Laboratories and Research, Empire State Plaza, Albany, NY 12201 .
}

$1983 a, b, c)$. Monkeys are able to change SSR amplitude when reward is made dependent on such change. SSR increase (SSR $\uparrow$ mode) or SSR decrease (SSR $\downarrow$ mode) occurs without change in initial muscle length, in the stretch eliciting the SSR, or in the background activity of agonist, antagonist, or synergist muscles. The most distinctive feature of SSR amplitude change, increase or decrease, is its time course. It occurs very slowly, becoming obvious only after 5 to 10 days, and progressing over at least 2 months. Reversal and redevelopment also occur very slowly.

Following these initial studies, and in preparation for further work, we combined all available data to produce composite SSR $\uparrow$ and SSR $\downarrow$ curves describing the development of SSR amplitude change over several months. We expected simply better definition of the slow development already apparent in the curves of individual animals. Instead, the composite curves, by averaging out random day-to-day variations and thus increasing the signal-to-noise ratio, reveal previously unexpected features of SSR amplitude change. They indicate that change occurs in two distinct phases. This finding adds to the body of evidence suggesting that the SSR also satisfies the third requirement, possession of the responsible substrate. 


\section{Materials and Methods}

Data collection. Animal preparation and data collection methods, described in detail elsewhere (Wolpaw and Seegal, 1982; Wolpaw et al., $1983 \mathrm{a}, \mathrm{b}$ ), are summarized here. Subjects were nine monkeys (Macaca nemestrina or $M$. mulatta, male, 5 to $7 \mathrm{~kg}$ ), prepared under general anesthesia with chronic intramuscular stainless steel fine-wire EMG electrodes in one biceps muscle (as well as in synergist and antagonist muscles). Wires passed through a protected exit in the forearm to a small connector plug. EMG was amplified ( $\times 1000$, bandpass 10 to $2000 \mathrm{~Hz}$ ) and digitized $(2000 \mathrm{~Hz})$.

Each monkey sat with forearm resting in a cast attached at the elbow to a torque motor shaft. Hand, forearm, and shoulder positions were fixed. The animal was taught by computer to maintain elbow angle at $90^{\circ}\left( \pm 1.5^{\circ}\right)$ against steady moderate extension torque $(0.5$ newton-meter $(\mathrm{Nm}))$ provided by the motor. If correct angle was held for a randomly selected 1.2- to 1.8-sec period and if the average absolute value of biceps EMG for the last 0.2 sec was within a specified range, the motor gave a short pulse of additional extension torque which briefly extended the elbow and elicited the biceps SSR. The computer digitized EMG and elbow angle following pulse onset, and calculated the average absolute value of biceps EMG in the SSR interval (typically 14 to $24 \mathrm{msec}$ after pulse onset). Liquid reward was given $200 \mathrm{msec}$ after pulse onset. Thus, the animal held correct elbow angle and correct biceps EMG for a randomly chosen period, felt the slight extension pulse, opened its mouth, and received the reward squirt. The task functioned under one of three modes. Under the control mode, reward always occurred following the pulse. Under the SSR $\uparrow$ or SSR $\downarrow$ mode, reward occurred only if the average absolute value of biceps EMG in the SSR interval was greater (SSR $\uparrow$ ) or less (SSR $\downarrow$ ) than a specified value.

Once trained to the control mode, monkeys normally completed 3000 to 6000 trials each day. The computer gave a summary every $3 \mathrm{hr}$, which included average background (i.e., prepulse) biceps EMG amplitude, average initial elbow angle, and average course of biceps EMG amplitude and elbow angle following pulse onset. It also provided a grand summary every $24 \mathrm{hr}$, with a histogram of the SSR amplitudes of all individual trials. Daily biceps SSR amplitude was defined as the average EMG amplitude in the SSR interval minus average background EMG amplitude.

Data were collected from each animal over 4 to 13 months. Throughout this time, background EMG and the initial $30 \mathrm{msec}$ of pulseinduced extension remained stable. For the first 7 to 30 days the animal worked under the control mode. Then, the animal was switched to the $\mathrm{SSR} \uparrow$ or SSR $\downarrow$ mode for up to 100 days. In the following months, most animals underwent mode reversal (SSR $\uparrow$ to SSR $\downarrow$ or vice versa), reexposure to the previous mode, and periods of nonperformance. We meticulously monitored animal well-being throughout the prolonged course of the studies. Food and water consumption, weight, skin condition, and demeanor were followed closely. Full details of animal care procedures have been published (Wolpaw and Seegal, 1982; Wolpaw et al., 1983b). All animals remained healthy and active throughout data collection.

Composite curves. The nine animals provided 18 curves of SSR change, 11 SSR $\uparrow$ curves and 7 SSR $\downarrow$ curves, each consisting of daily SSR amplitudes following mode switch. These included initial development curves from each animal, and reversal and redevelopment curves from animals exposed to more than one mode switch. (Reversal and redevelopment curves follow courses indistinguishable from initial development curves (Wolpaw, 1983).) All SSR $\downarrow$ curves and eight SSR $\uparrow$ curves extended 40 to 100 days following mode switch. The latter parts of these curves were broken by 10 - to 12 -day periods of nonperformance (Wolpaw et al., 1983b). The other three SSR $\uparrow$ curves extended 36, 21, and 20 days.

Earlier studies had determined the average magnitude of SSR amplitude change. The reason for generating composite SSR $\uparrow$ and SSR $\downarrow$ curves in the present study was to better define the development of SSR amplitude change, the characteristic shapes of the curves from the first hours after mode switch to the end of data collection months later. To give maximum definition of the course of change, and to give each individual curve equal weight, the following three steps were taken with each individual curve prior to combining them. (1) A control SSR amplitude was obtained by averaging the daily SSR amplitudes for the 10 days preceding mode switch. (2) This control SSR amplitude was subtracted from the SSR amplitude for each post-swilch day, giving $\Delta$, the difference from control for each day. (3) Each day's $\Delta$ was divided by the average $\Delta$ for the days 36 to 40 after mode switch and multiplied by 100 , giving each day's $\Delta$ as percentage of $\Delta$ achieved at 36 to 40 days. This normalization step permitted the composite curve to define the average course of SSR amplitude change largely free of the obscuring effects of the variation among individual animals in absolute amplitude of SSR amplitude change finally achieved (Wolpaw et al., 1983b). (Ideally, SSR amplitude change would have been completed before the days used for this normalization. The 36- to 40-day period was the latest period for which all but three of the individual curves had data and, in fact, most SSR change had already occurred (see Fig. 2).) For each SSR $\uparrow$ curve lacking 36- to 40-day data, the average $\Delta$ for the last 3 days available was calculated. This value and the $\Delta$ 's for the same days from the individual curves which did extend to 40 days were used to determine a projected 36- to 40 -day $\Delta$ for the short curve.

The normalized individual curves generated by these three steps were then combined to give a mean $\Delta$ and its SEM for each day following mode switch. The available data allowed calculation out to 78 days for the composite SSR $\uparrow$ curve and 59 days for the composite SSR\ curve. Because data collection stopped, or was interrupted, in the latter parts of individual curves, there were fewer individual values (as few as three) for each day past 40 days. Thus, the later mean $\Delta^{\prime} s$ have larger SEMs. For these post-40-day data, the daily $\triangle$ 's and SEMs were averaged to give values for each 2-day period. All of the composite $\Delta$ 's (and their SEMs) were converted from percentages of the 36- to 40 -day $\Delta$ 's to percentages of control SSR amplitude and 100 was added to each value. Thus, Figure 2 shows mean post-switch SSR amplitudes as percentages of control SSR amplitude and illustrates not only the course of SSR amplitude change but its average magnitude as well.

Using the 3-hr summaries, analogous procedures provided 0 - to 6 $\mathrm{hr}, 6-$ to $12-\mathrm{hr}$, and 12 - to $24-\mathrm{hr}$ composite values for the first day of SSR amplitude change. (The relative paucity of 12 - to 18 -hr data (12:00 midnight to 6:00 AM, since mode switch occurred at noon) necessitated its combination with the 18- to 24-hr data.) The obscuring effects of the normal diurnal rhythm (Wolpaw and Seegal, 1982) were eliminated, or at least markedly reduced, by using the 2 days preceding mode switch to provide an estimate of the rhythm, and subtracting this estimate out of the composite data. (The diurnal rhythm is little affected by adaptive SSR change (Wolpaw et al., 1984). Thus this procedure is justified.)

\section{Results}

SSR amplitude changed appropriately following each of the 18 mode changes. Figure 1 shows representative samples of change produced following mode switch. The animal in Figure $1 A$ shows steady increase in SSR amplitude following onset of the SSR $\uparrow$ mode. The five graphs give absolute value of biceps EMG and average elbow extension for $40 \mathrm{msec}$ following pulse onset for the days indicated. Each graph is the average of the full day's data (3000 to 6000 trials). Also shown are raw EMG traces from three consecutive single trials on day 0 and day 80 . Whereas background EMG, represented here by the first 10 msec following pulse onset, and elbow extension remain stable, SSR amplitude undergoes a marked increase. (The effect of SSR amplitude change on elbow extension does not begin until about $40 \mathrm{msec}$, due to the time necessary for motor unit contraction following excitation (Wolpaw et al., 1983b).) Figurc $1 B$ displays comparable data from another monkey following onset of the SSR $\downarrow$ mode. Steady decrease in SSR amplitude occurs without change in background EMG or elbow extension.

Composite curves. Figure 2 shows the composite SSR $\uparrow$ and SSR $\downarrow$ curves. The first three points of each curve are hr 0 to 6 , 6 to 12 , and 12 to 24 of the first day. For days 2 to 40 , daily values are shown; beyond 40 days, 2 -day values are shown. The inset of Figure 2 is a magnified view of the first week. Each curve shows a nearly immediate mode-appropriate offset followed by a gradual appropriate change which continues at a slowly declining rate indefinitely. The inset indicates that this offset is present after $6 \mathrm{hr}$. Both composite curves appear to be approaching asymptotes and to approximate hyperbolas. The empirically selected equation $y=\frac{a}{b+1 / x}+c$ was fit to each curve by a computer program which minimized the sum of the 

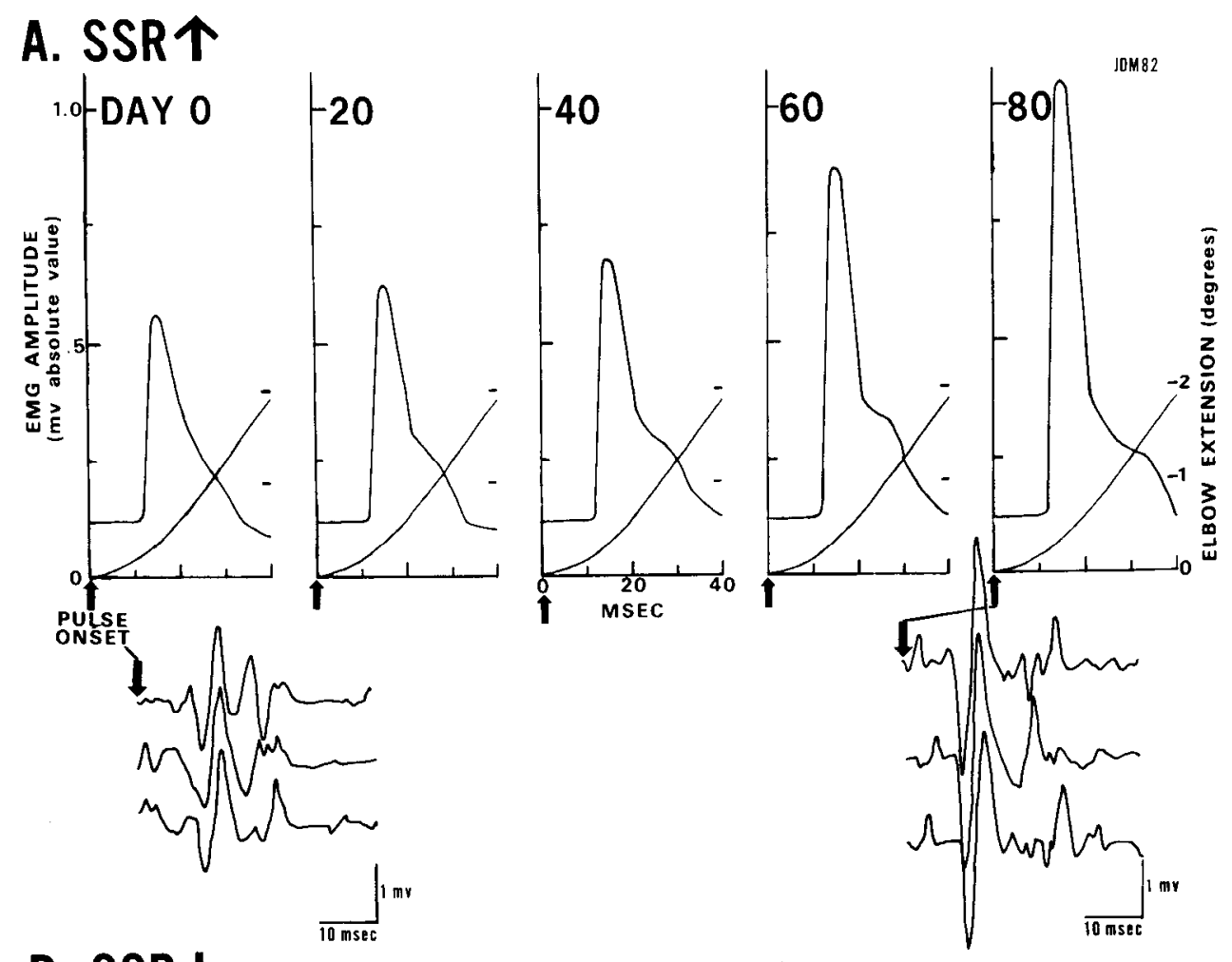
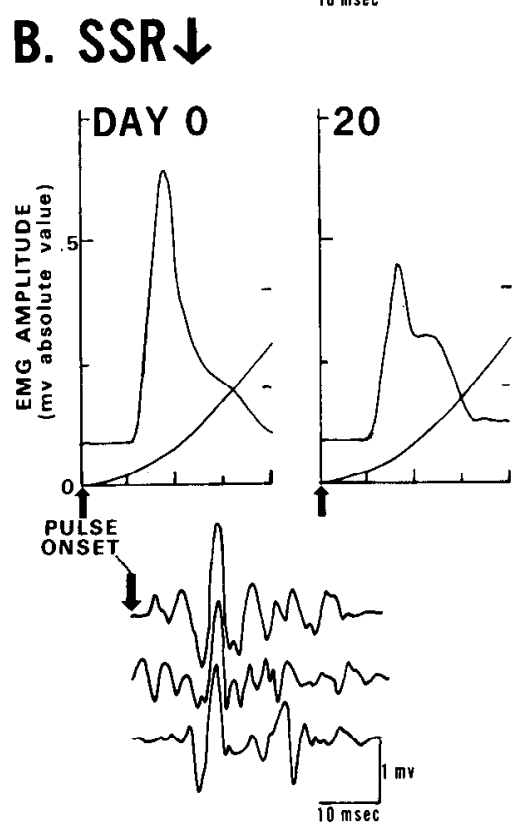

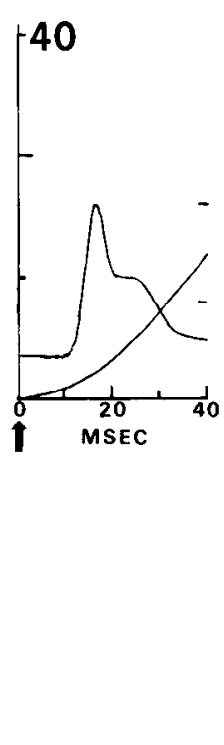

RAR823

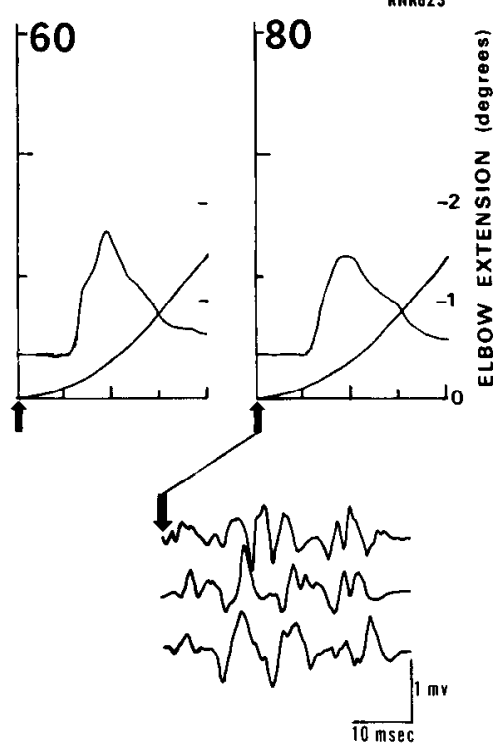

Figure 1. A, The five graphs show average daily SSR amplitude and elbow extension for $40 \mathrm{msec}$ following pulse onset for representative days beginning with imposition of the SSR $\uparrow$ mode in one monkey. Each trace comprises the full day's data (3000 to 6000 trials). Note the steady increase in SSR amplitude without change in background EMG (represented here by the first $10 \mathrm{msec}$ following pulse onset) or in elbow extension. The two sets of traces below show representative consecutive single trials of raw EMG on days 0 and 80 and illustrate the marked increase in SSR amplitude eventually produced. $B$, Analogous data from another monkey exposed to the SSR $\downarrow$ mode. Progressive decrease in SSR amplitude occurs without change in background EMG or in the first 40 msec of pulse-induced extension.

squares of the residuals, using an iterative, modified, steepest descent procedure. The resulting lines are superimposed on the data in Figure 2. For the SSR $\uparrow$ curve, optimum values are $a=$ $1.71, b=0.01$, and $c=108.31$. For the SSR $\downarrow$ curve, optimum values are $a=-2.99, b=0.071$, and $c=93.06$. The asymptotes of these lines provide estimates of the total SSR change to be expected if data collection continued indefinitely. The SSR $\uparrow$ curve approaches $279 \%$ of control amplitude, and the SSR $\downarrow$ curve approaches $51 \%$. Thus, for the SSR $\uparrow$ curve, the projected final change from control is $+179 \%$, whereas, for the SSR $\downarrow$ curve it is $-49 \%$.

Phases $I$ and $I I$. The most striking and unexpected feature 


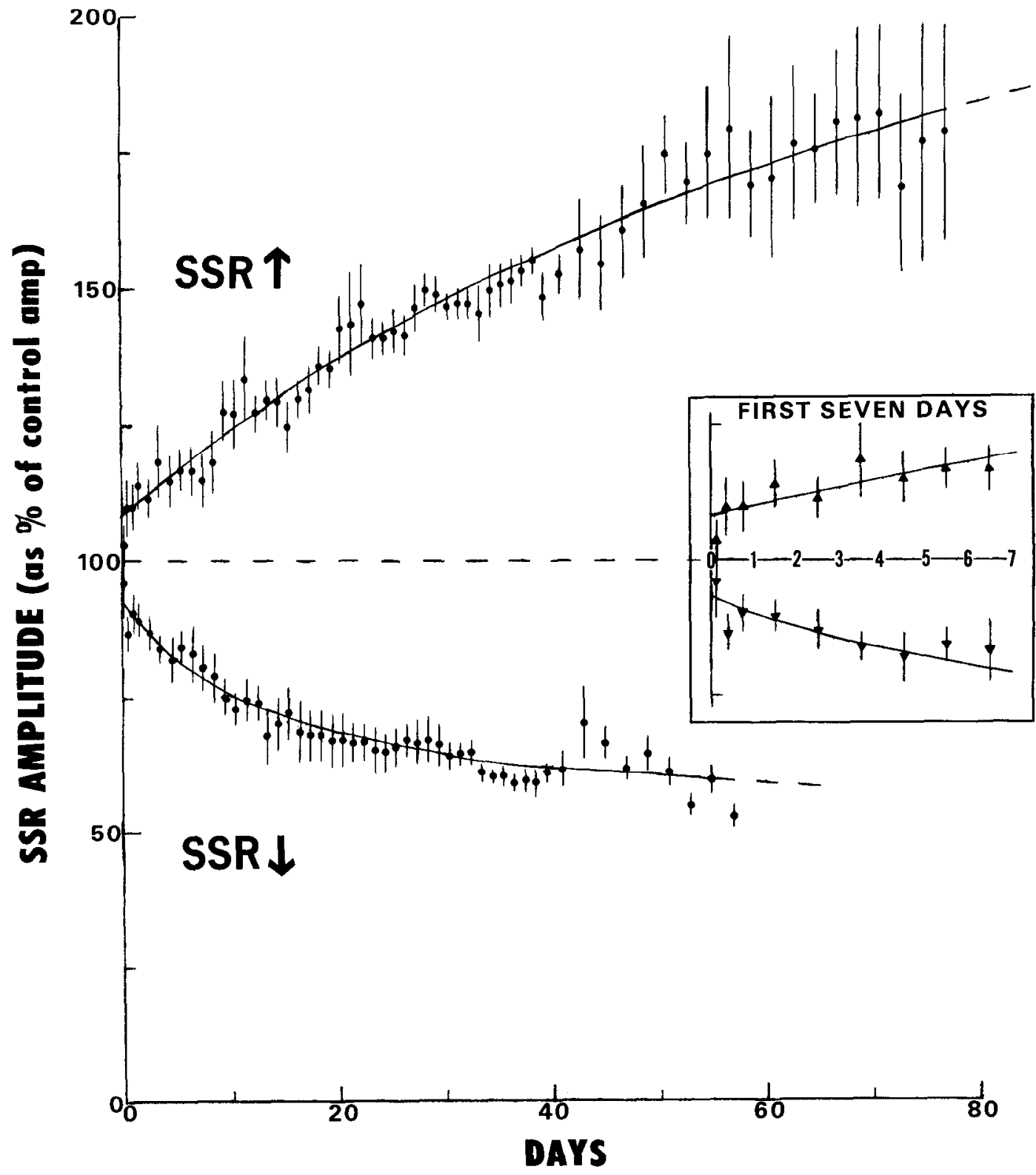

Figure 2. Composite plots of SSR amplitude under the SSR $\uparrow$ mode and under the SSR $\downarrow$ mode from 18 mode switches (11 SSR $\uparrow$ and 7 SSR $\downarrow$ ) in nine monkeys. Each plotted value is the average SSR amplitude ( \pm SEM) as a percentage of control SSR amplitude. Values are given for $\mathrm{hr} 0$ to 6,6 to 12 , and 12 to 24 of the first day, for each subsequent day through 40 days, and for every 2 days from then on. The inset is a horizontally expanded view of the first week. See the text for details of calculations. The superimposed lines are computer fits of the equation $y=\frac{a}{b+1 / x}+c$. Each curve shows a nearly immediate (within $6 \mathrm{hr}$ ) mode-appropriate offset followed by slow change which continues indefinitely.

of each composite curve is the initial appropriate offset, clearly present by $6 \mathrm{hr}$ after mode switch. For both SSR $\uparrow$ and SSR $\downarrow$ modes, amplitude change appears to occur in two phases, a nearly immediate change followed by much slower change which continues indefinitely. We termed the initial rapid change phase I and the subsequent slow change phase II, and tested this two-phase hypothesis in several ways.

First, we evaluated the importance of the constant, $c$, to the goodness-of-fit achieved by the equation $y=\frac{a}{b+1 / x}+c$. The initial offset, or the hypothesized phase I, is represented by $c-$ 100. To test its importance, the computer compared the good- ness-of-fit of the original equation, $y=\frac{a}{b+1 / x}+c$, with that of $y=\frac{a}{b+1 / x}+100$, which does not provide for an initial offset. ${ }^{3}$ The equation containing $c$ was clearly better $(p<<$ $0.001)$ for both the SSR $\uparrow$ curve $\left(F_{1,58}=25.9\right)$ and the SSR $\downarrow$ curve $\left(F_{1,48}=20.4\right)$. Thus, these results support the existence of phase I.

\footnotetext{
${ }^{3}$ In order to maximize the sensitivity of the procedure, 100 was subtracted from each side of each equation prior to the computer comparison.
} 
In spite of the striking nature of the composite curves and the support offered by the fitted equations, the two-phase hypothesis can still be questioned on several grounds. First, the evidence for phase I, the nearly immediate mode-appropriate offset, might be fortuitous, a chance result of the normal dayto-day variations. Second, if we accept phase I, it is then possible to question phase II. Phase II change appears slow and continuous in the composite curves of Figure 2. However, on the basis of these curves alone, it is possible that phase II actually consists of multiple phase I-like jumps obscured in the individual curves by the normal day-to-day variations. By this interpretation, the first phase I change in each individual curve occurs on the first day and thus is evident in the composite curves, whereas subsequent phase I changes occur on different days in different individual curves and thus are not evident in the composites. To test both of these possibilities - that the initial offsets comprising phase I were chance variations and that phase II consisted of multiple phase I-like jumps-we returned to the individual curves used to generate the composite curves of Figure 2 and evaluated the day-to-day changes.

First, to evaluate phase II, we determined for each day of each individual curve, except the first day, the SSR amplitude change from the preceding day. This day-to-day difference value was expressed as percentage of the control SSR amplitude of the individual curve. The difference predicted for that day by the slope of the computer-generated equation in Figure 2 (i.e., the expected slow, or phase II, change) was then subtracted to give the residual change. Finally, to remove from the residual the distorting effect of the progressive change occurring in SSR amplitude over the course of mode exposure, it was converted to percentage of the 2-day average SSR amplitude (i.e., average amplitude for the 2 days used to calculate the residual). We obtained 573 such residuals from the $\mathrm{SSR} \uparrow$ data and 330 values from the SSR $\downarrow$ data. The histograms in Figure 3 show their distributions. Both appear unimodal. The means are very close to zero (as expected due to the subtraction of expected phase II change) and the SDs are similar $(-0.1 \pm 7.1$ (0.3 SEM) for the SSR $\uparrow$ data and $0.0 \pm 7.5(0.4 \mathrm{SEM})$ for the SSR $\downarrow$ data).

If phase II is in fact the slow continuous change indicated in the composite curves, the procedure described above should have eliminated the effects of the SSR $\uparrow$ or SSR $\downarrow$ mode on the post-first day difference values. The distributions of SSR $\uparrow$ and SSR $\downarrow$ residuals should be indistinguishable from each other. If, on the other hand, phase II actually consists of multiple phase I-like jumps, the SSR $\uparrow$ and SSR $\downarrow$ distributions should be mirror images, or at least distinctly different from each other. Each should be bimodal or have a prominent shoulder, indicating the presence of a distinct subpopulation comprising $\sim 15 \%$ of the values. For the SSR $\uparrow$ distribution, this subpopulation, representing phase I-like SSR increases, should be clearly to the right of zero and the major distribution should be slightly to the left of zero. In contrast, for the SSR $\downarrow$ distribution, this subpopulation, representing phase I-like decreases, should be clearly to the left of zero and the major distribution should be slightly to the right of zero. However, as Figure 3 shows, the $\mathrm{SSR} \uparrow$ and SSR $\downarrow$ distributions are very similar. They appear to be unimodal about zero and, most important, show no evidence of the dissimilarity to be expected if phase II consists of multiple phase I-like jumps. This apparent similarity was confirmed by the Kolmogorov-Smirnov two-sample test (Siegal, 1956) which revealed no significant difference $(p>0.30)$ between the two distributions. These results indicate that phase II change is slow and continuous, as the composite curves suggest, and thus is definitely different from phase I.

The day-to-day differences of the individual curves also offer an opportunity to evaluate the evidence for phase I. The dayto-day residuals for day 1 , which are not included in the histograms of Figure 3, should be clearly different from the

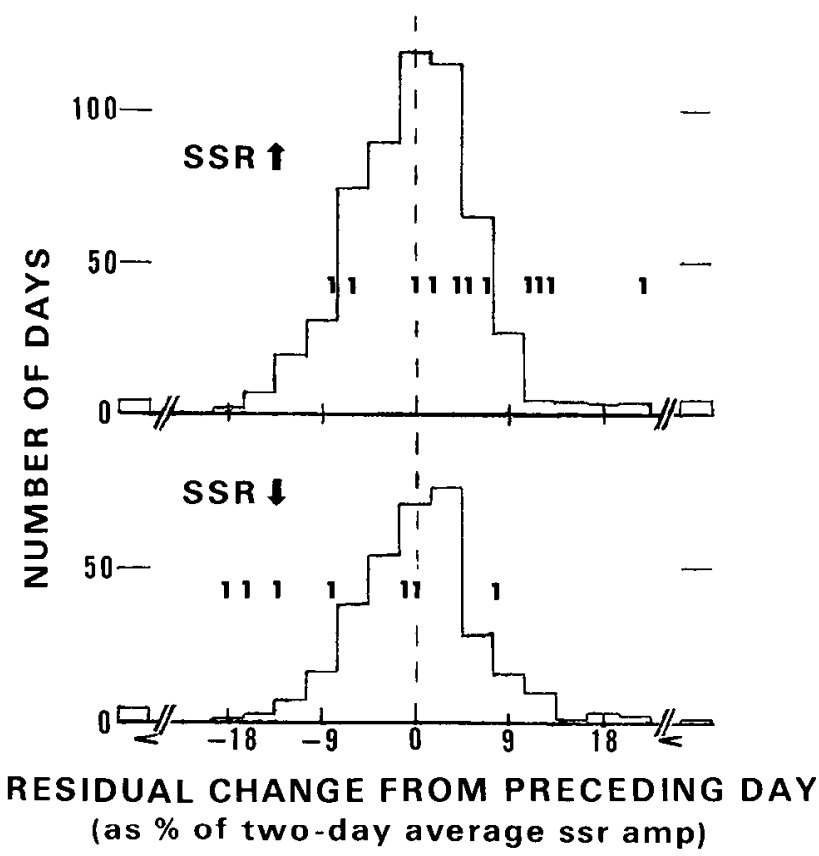

Figure 3. Histograms of residuals (difference from preceding day's SSR amplitude minus change predicted by the slope of the computerfitted equation) for each day, except the first day, of each individual SSR $\uparrow$ or SSR $\downarrow$ curve, expressed as percentage of the 2-day average SSR amplitude. The SSR $\uparrow$ and SSR $\downarrow$ histograms appear to be unimodal about zero and are very similar. The superimposed 1's are the first day residuals. They are shifted significantly $(p<0.01)$ to the right for the $\mathrm{SSK} \uparrow$ data and significantly $(p<0.025)$ to the left for the SSR $\downarrow$ data. See the text for full details.

post-first day residuals making up the histograms. We calculated for each individual curve the difference between SSR amplitude for the $24 \mathrm{hr}$ prior to mode switch and SSR amplitude 6 to $30 \mathrm{hr}$ after mode switch (i.e., after the presumed occurrence of phase I). This first day difference value was treated just as described above for the post-first day difference values: it was expressed as percentage of the control SSR amplitude of the individual curve; the difference predicted by the slope of the computer-generated equation (i.e., the difference expected if the change occurring in the first day was simply phase II change) was subtracted; and, finally, the residual was converted to percentage of the 2-day average SSR amplitude. The first day residuals thus calculated are shown by the 1's superimposed on the Figure 3 histograms. As expected, their variability, due to normal day-to-day variations, is comparable to that of the post-first day residuals. To support the existence of phase I, their means should be different from those of the post-first day residuals. The SSR $\uparrow$ first day residuals should be shifted to the right and the SSR $\downarrow$ residuals to the left, indicating the presence of mode-appropriate phase I jumps. This is the case. The SSR $\uparrow$ first day residuals have a mean of $+5.6 \pm 8.7 \mathrm{SD}(2.8 \mathrm{SEM})$, which is appropriately shifted ( $p<0.01$ by the Mann-Whitney $U$ test (Siegal, 1956)). The residuals for each subsequent day (days $2,3, \ldots 78$ ) were similarly tested and failed to show a shift of comparable significance. The SSR first day residuals have a mean of $-6.7 \pm 9.7 \mathrm{SD}$ (3.9 SEM), which is also appropriately shifted $(p<0.025) .{ }^{4}$ Again, none of the subsequent days showed a comparable shift. Thus, these results support phase I and the two-phase hypothesis.

\footnotetext{
${ }^{4}$ We used the Mann-Whitney $U$ test since it does not assume that the tested populations are normally distributed. The $t$ test, which does assume normality, yielded comparable values (SSR $\uparrow$ first day residuals: $t=2.59, p<0.01 ; \mathrm{SSR} \downarrow$ first day residuals: $t=2.15, p<0.02$ ).
} 
TABLE I

Comparison of phase I and phase II in regard to rates of SSR amplitude change, final magnitudes of SSR amplitude change achieved (or predicted), and rates of reward increase

\begin{tabular}{lccccc}
\hline & \multicolumn{2}{c}{ Phase I } & & \multicolumn{2}{c}{ Phase II } \\
\cline { 2 - 3 } \cline { 5 - 6 } & $\mathrm{SSR} \uparrow$ & $\mathrm{SSR} \downarrow$ & & $\mathrm{SSR} \uparrow$ & $\mathrm{SSR} \downarrow$ \\
\hline $\begin{array}{c}\text { SSR amplitude } \\
(\Delta / \text { day })\end{array}$ & $+35 \%$ & $-31 \%$ & & $+1.7 \%$ & $-2.4 \%$ \\
$\begin{array}{c}\mathrm{SSR} \text { amplitude } \\
\quad(\text { final } \Delta)\end{array}$ & $+8.7 \%$ & $-7.7 \%$ & & $(+170 \%)$ & $(-41 \%)$ \\
$\begin{array}{c}\text { Rewards } / 100 \text { trials } \\
(\Delta / \text { day })\end{array}$ & +50 & +44 & & +2.4 & +3.4 \\
\hline
\end{tabular}

Phase I and phase II magnitudes and rates. The value of the fitted equation at $6 \mathrm{hr}$ after mode onset provides an estimate of the magnitude of phase I change. For the SSR $\uparrow$ data, the estimate of phase I change is $+8.7 \%$. For the SSR $\downarrow$ data it is $-7.7 \%$. These estimates, subtracted from the estimates of total SSR amplitude change provided by the asymptotes of the fitted curves $(+179 \%$ for SSR $\uparrow$ and $-49 \%$ for SSR $\downarrow)$ furnish estimates of phase II magnitude. The estimated SSR $\uparrow$ phase II change is $+170 \%$. The estimated SSR $\downarrow$ phase II change is $-41 \%$. Thus, although phase II is much slower than phase I, its final magnitude is far greater.

The magnitude estimates provided above and the assumption that phase I occurs in $6 \mathrm{hr}$ furnish estimates of phase I rates: $+35 \%$ /day for the SSR $\uparrow$ curve and $-31 \%$ /day for the SSR $\downarrow$ curve. Initial, maximum, phase II rates may be estimated by taking the slopes of the fitted plots for day 1-2 after mode switch. In contrast to the phase I rates, the maximum SSR $\uparrow$ phase II rate is $+1.7 \%$ /day, and the maximum SSR $\downarrow$ phase II rate is $-2.4 \% /$ day. Thus the phase $\mathrm{I}$ rate is 21 times the phase II rate for the SSR $\uparrow$ data and 12 times the phase II rate for the SSR data. Table I presents these phase I and phase II magnitude and rate estimates to facilitate their comparison.

Phase I and phase II reward increase rates. These rate estimates and the histograms of SSR amplitude for all individual trials given each day by the computer (Wolpaw et al., 1983b) allow calculation of the rates of reward percentage increase provided by phase I and phase II. The reward increase rates for $\mathrm{SSR} \uparrow$ and SSR $\downarrow$ phase I change are $50 \%$ /day and $44 \%$ /day, respectively. For example, an animal receiving rewards on 50 of each 100 trials in the first minutes after the switch to the $\mathrm{SSR} \uparrow$ mode would receive rewards on 62 to 63 of 100 trials 6 hr later, after phase I change had taken place. In contrast, the reward rate increases for SSR $\uparrow$ and SSR $\downarrow$ phase II changes are at most $2.4 \%$ /day and $3.4 \%$ /day, respectively. Thus, an animal exposed to the SSR $\uparrow$ mode and undergoing phase II change would receive only 2 to 3 additional rewards per 100 trials after $24 \mathrm{hr}$. Table I facilitates comparison of these phase I and phase II reward increase rates.

\section{Discussion}

Initial studies (Wolpaw et al., 1983a, b) indicated that SSK amplitude change occurred gradually, progressing over weeks and months. The normal day-to-day variations in SSR amplitude made it difficult to confidently identify in individual animals any early effects of the SSR $\uparrow$ or SSR $\downarrow$ mode. The accumulation of data and the finding that reversal and redevelopment curves do not differ from initial development curves (Wolpaw, 1983) allowed construction of the composite curves shown in Figure 2. Each curve shows a nearly immediate offset, followed by much slower prolonged change. This interpretation is supported by the expanded view of the initial week, by computer evaluation of the fitted equations, and by statistical analysis of the day-to-day changes in the individual curves making up the composites. Thus, SSR amplitude change, either increase under the SSR $\uparrow$ mode or decrease under the SSR $\downarrow$ mode, appears to occur in two phases. Phase I change, an estimated $8.7 \%$ increase (SSR $\uparrow$ ) or $7.7 \%$ decrease (SSR $\downarrow$ ) in SSR amplitude, occurs within $6 \mathrm{hr}$. Much slower phase II change occurs at a gradually declining rate over at least 2 months. Phase II rates are, at most, 5 to $8 \%$ of phase I rates; however, phase II eventually produces SSR amplitude change many times that produced by phase I.

On the most elementary level, these results demonstrate that the slow course of SSR amplitude change revealed by the initial studies cannot be explained by relatively trivial factors, such as animal failure to recognize or properly respond to the requirements of the task. The nearly immediate phase I change is clear evidence that animals respond appropriately to the SSR $\uparrow$ or SSR $\downarrow$ mode very quickly. The major implications of the composite curves derive from the clear separation of SSR amplitude change into two phases and concern the relationship between the two phases.

Phase I. Because the SSR is a purely segmental and largely monosynaptic phenomenon, the mechanism of phase I change in SSR amplitude is certainly present at the segmental level, probably in the monosynaptic arc of the SSR. As discussed in detail elsewhere (Wolpaw et al., 1983b), the most likely candidate is change in Ia synaptic function, perhaps mediated presynaptically, and the next most likely is change in muscle spindle function, probably mediated by gamma motoneurons. The mechanism is presumably activated by altered activity in descending spinal cord pathways. For example, altered activity in corticospinal, reticulospinal, or vestibulospinal pathways could alter presynaptic inhibition at the Ia synapse (see Baldissera et al., 1981, for review) and thereby change alpha motoneuron response to the Ia afferent volley produced by the extension pulse. ${ }^{5}$ Because pulse onset time is unpredictable (see "Materials and Methods") and the SSR occurs before any other possible response, the change in descending spinal cord activity must be tonic; it must be present day after day, for a considerable portion of the 5 to $7 \mathrm{hr} /$ day the animal spends at the task. As the discussion below indicates, this conclusion concerning phase I change seems crucial to understanding the genesis of phase II change.

The rapidity of phase I change, on initial mode switch as well as on subsequent switches, ${ }^{6}$ indicates that it does not depend on persistent alteration, segmental or suprasegmental. Thus, the term "plasticity," as it is normally used to connote long-term persistent change, with a structural or biochemical basis, does not seem applicable to phase I.

Phase II. Like phase I, phase II indicates change in the function of the segmental reflex arc. Its mechanism could be gradual further change in the tonic descending activity imping-

\footnotetext{
${ }^{5}$ Given the rapid occurrence and relatively small magnitude of phase I change, its mechanism could also be a relatively nonspecific result of changes in descending activity. For example, a very subtle change in arm posture could conceivably alter muscle spindle response to the extension pulse. It is also conceivable that the phase I change is a product of extraneuronal, humoral alterations, such as change in circulating sympathetic agents, affecting the muscle spindle or another station in the SSR arc. However, the relative specificity of phase II change, at least, to the agonist muscle (Wolpaw et al., 1983c), its large magnitude, and the possible causal relationship between phase I and phase II discussed below, make nonspecific neuronal or extraneuronal mechanisms for phase I unlikely.

${ }^{6}$ Comparison of the individual initial development curves with the individual reversal and redevelopment curves revealed no significant differences in the magnitude or rapidity of phase I change.
} 
ing on the segmental apparatus, or gradual alteration in the segmental reflex apparatus itself, altering the effect of the descending activity. At present, the critical question concerning phase II arises from its extremely slow rate.

Under the control mode, when SSR amplitude does not matter, the descending activity controlling the reflex arc presumably varies randomly over a considerable range. Once the mode is switched to SSR $\uparrow$ or SSR $\downarrow$, reward percentage becomes dependent on location within this range. As the phase I change shows, the animal quickly begins to maintain tonic descending activity in the more highly rewarded portion of the available range. It is guided to this portion by the substantial and rapid increase in reward percentage achieved. For example, an animal confronted by the SSR $\uparrow$ mode will achieve about 62 rewards/ 100 trials if it operates in the SSR $\uparrow$ portion of the range, about 50 if it continues, as under the control mode, to operate over the entire range, and only about 38 if it operates in the SSR $\downarrow$ portion of the range. Thus phase $I$ is readily explicable as an operantly conditioned phenomenon, provoked by the reward contingency. In contrast to the phase I situation, the reward percentage increase provided by phase II change on an hourto-hour or even day-to-day basis is minimal, far smaller even than the effects on reward percentage of the diurnal rhythm in SSR amplitude (Wolpaw and Seegal, 1982). At its most rapid rate, phase II provides about 3 extra rewards per 100 trials, spread over $24 \mathrm{hr}$ and obscured by diurnal and other variations in SSR amplitude. This tiny increase hardly seems significant positive feedback. ${ }^{7}$ Why then does phase II change occur and continue over months?

It is still conceivable that phase II is operantly conditioned, despite the minimal reinforcement it provides. However, a more reasonable explanation appears to be that phase II change is a long-term result of the alterations in tonic descending activity responsible for phase I and needed to maintain phase I. The continued presence of this altered tonic activity, day after day, may produce structural and/or biochemical alterations which alter the function of the segmental reflex arc. Such alterations could be located suprasegmentally, perhaps in the cerebellum, which has considerable influence over the segmental reflex arc and has been implicated as a site of other adaptive changes (see Thompson et al., 1983, for review). Or they could be located at the segmental level, either in the arc of the SSR, perhaps in the Ia synaptic region, or at some site in the segmental apparatus exerting influence over the SSR.

Considerable laboratory and clinical evidence strongly suggests that long-term continual descending influence may produce enduring alterations in the spinal cord, alterations which persist without continued impetus from above. This body of evidence includes the studies of DiGiorgio (1929) and others (Alella, 1948; Manni, 1950; Chamberlain et al., 1963) demonstrating that the segmental effects of various suprasegmental lesions persist long after cord transection. It includes clinical and laboratory studies describing the changes which occur in segmental function in the weeks and months following traumatic cord transection (Brown-Sequard, 1879; McGough, 1924; Nelson and Mendell, 1979; Mountcastle, 1980; Naftchi, 1982; Mendell, 1984). These studies showing the cord's capacity for enduring change are supported by studies of the last several decades demonstrating that the isolated spinal cord can be classically conditioned (see Patterson, 1980, for review).

\footnotetext{
${ }^{7}$ The daily histograms of the SSR amplitudes of individual trials (Wolpaw et al., 1983 b) provided no evidence that the small daily phase II change was due to marked change in a small proportion of the day's trials. Rather, it appeared to be due to slight change in the SSR of each trial.
}

For example, suppose that phase I change was mediated by neurons synapsing on the Ia terminals and producing presynaptic inhibition. SSR $\downarrow$ phase I change would then be due to increased firing of these neurons and consequent increased presynaptic inhibition. As noted above, the firing increase would of necessity be a tonic increase, an increase present continually for many hours each day. It seems quite likely that such steady-state change would eventually produce biochemical or even structural changes in the presynaptic contact or in the Ia synapse itself. It would probably be more surprising if no change occurred.

Whether phase II is due to suprasegmental and/or segmental alteration, the conclusion that it is a long-term, persistent result of the altered tonic descending activity responsible for phase I is difficult to avoid. Phase II probably reflects structural and/ or biochemical alterations and may be quite properly labeled "plasticity." If persistent segmental alteration underlies phase II change, such alteration should constitute a technically accessible substrate of memory.

\section{References}

Alella, A. (1948) Persistence of asymmetries of cerebral origin in the extremities of animals with cord section. Arch. Fisiol. 47: 105-112.

Baldissera, F., H. Hultborn, and M. Illert (1981) Integration in spinal neuronal systems. In Handbook of Physiology. Sect. I: The Nervous System. Vol. II: Motor Control. Part I, V. B. Brooks, ed., pp. 509595 , Williams \& Wilkins, Baltimore.

Brown-Sequard, C. E. (1879) Recherches experimentales sur une nouvelle propriete du system nerveux. C. R. Hebd. Seances Acad. Sci. 89: $889-913$.

Chamberlain, T. J., P. Halick, and R. W. Gerard (1963) Fixation of experience in the rat spinal cord. J. Neurophysiol. 26: 662-673.

DiGiorgio, A. M. (1929) Persistenza nell'animale spinale, di asymmetrie posturali e motorie di origine cerebellare. Arch. Fisiol. 27: 518-580.

Manni, E. (1950) Localizzazoni cerebellari corticali nella cavia. Nota I: I1 "corpus cerebelli." Arch. Fisiol. 49: 213-237.

McGough, G. P. (1924) The relation of the pyramidal tract to spinal shock. Am. J. Physiol. 71: 137-152.

Mendell, L. M. (1984) Modifiability of spinal synapses. Physiol. Rev. 64: $260-324$.

Mountcastle, V. B. (1980) Effects of spinal cord transection. In Medical Physiology, V. B. Mountcastle, ed., Vol. 1, pp. 781-786, C. V. Mosby Co., St. Louis.

Naftchi, N. E. (1982) Spinal Cord Injury, Spectrum, New York.

Nelson, S. G., and L. M. Mendell (1979) Enhancement in Ia-motorneuron synaptic transmission caudal to chronic spinal cord transection. J. Neurophysiol. 42: 642-654.

Patterson, M. M. (1980) Mechanisms of classical conditioning of spinal reflexes. In Neural Mechanisms of Goal-Directed Behavior and Learning, R. F. Thompson, L. H. Hicks, and V. B. Shvyrkov, eds., pp. 263272, Academic Press, Inc., New York.

Siegal, S. (1956) Nonparametric Statistics for the Behavioral Sciences, pp. 116-136, McGraw-Hill, New York.

Thompson, R. F., T. W. Berger, and J. Madden, IV (1983) Cellular processes of learning and memory in the mammalian CNS. Annu. Rev. Neurosci. 6: 447-491.

Wolpaw, J. R. (1983) Adaptive plasticity in the primate spinal stretch reflex: Reversal and re-development. Brain Res. 278: 299-304.

Wolpaw, J. R., and R. F. Seegal (1982) Diurnal rhythm in the spinal stretch reflex. Brain Res. 244: 365-369.

Wolpaw, J. R., V. A. Kieffer, R. F. Seegal, D. J. Braitman, and M. G. Sanders (1983a) Adaptive plasticity in the spinal stretch reflex. Brain Res. 267: 196-200.

Wolpaw, J. R., D. J. Braitman, and R. F. Seegal (1983b) Adaptive plasticity in the primate spinal stretch reflex: Initial development. J. Neurophysiol. 50: 1296-1311.

Wolpaw, J. R., R. F. Seegal, and J. A. O'Keefe (1983c) Adaptive plasticity in the primate spinal stretch reflex: Behavior of synergist and antagonist muscles. J. Neurophysiol. 50: 1312-1319.

Wolpaw, J. R., P. A. Noonan, and J. A. O'Keefe (1984) Adaptive plasticity and diurnal rhythm in the primate spinal stretch reflex are independent phenomena. Brain Res. 300: 385-391. 